\title{
Pimavanserin for Psychosis in Parkinson's Disease-Related Disorders: A Retrospective Chart Review
}

\author{
Jessie Sellers ${ }^{1} \cdot$ R. Ryan Darby ${ }^{1} \cdot$ Alma Farooque $^{1} \cdot$ Daniel O. Claassen $^{1}$
}

Published online: 29 March 2019

(c) The Author(s) 2019

\begin{abstract}
Background Psychosis is common in Parkinson's disease-related disorders and is associated with significant morbidity. Pimavanserin is a newly approved treatment for Parkinson's disease psychosis, but real-world experience with pimavanserin has been limited by small sample sizes and limited assessment of longitudinal outcomes.

Objective The aim was to summarize the clinical experience with pimavanserin in a large cohort of patients with Parkinson's disease-related psychosis.

Methods We conducted a retrospective chart review of patients who were prescribed pimavanserin at Vanderbilt University Medical Center in the southeast United States between May 2016 and July 2018. We used Chi-squared analyses to compare efficacy and tolerability of pimavanserin, considering patient diagnosis, presence of dementia or delusions, use of deep brain stimulation, and prior antipsychotic failure. Additionally, we compared the clinical characteristics of patients who started treatment and those who did not, to evaluate safety outcomes.

Results We identified 107 patients prescribed pimavanserin, and 91 began treatment. Clinical improvement in psychosis was documented in 76\% of patients (69/91) and did not differ based on diagnosis, presence of dementia, delusions, use of deep brain stimulation, or prior antipsychotic failure. Adverse effects were reported in 20 patients (22\%), the most common of which was worsening gait instability $(5 / 91,5 \%)$. Side effects led to cessation of therapy in 11 of the 91 patients $(12 \%)$. At current follow-up, 50 (65\%) of 77 living patients remain on treatment, with a mean treatment duration of 14.6 months. Although most of these patients are on pimavanserin monotherapy (33/50,66\%), 17 patients (34\%) are on a dual-antipsychotic regimen. The living patients no longer on treatment stopped pimavanserin primarily because of a lack of perceived benefit $(11 / 77,14 \%)$, side effects $(9 / 77,12 \%)$, or both $(1 / 77,1 \%)$, though six patients $(8 \%)$ stopped for reasons unrelated to medication effects, including the desire to reduce overall medication burden and negative media reporting on pimavanserin. Conclusions Study results emphasize long-term efficacy and tolerability of pimavanserin for psychosis in Parkinson's diseaserelated disorders, including patients with dementia, delusions, deep brain stimulation use, or prior antipsychotic failure.
\end{abstract}

\section{Introduction}

Psychosis is a common non-motor symptom of Parkinson's disease (PD), causing substantial morbidity and increased mortality [1]. Psychosis prevalence in PD ranges from 43 to $60 \%$ [2], with even higher rates $(75 \%)$ in PD patients with dementia [3]. Visual hallucinations are most common, though other hallucinations (e.g., auditory, tactile)

Daniel O. Claassen

daniel.claassen@vumc.org

1 Department of Neurology, Division Behavioral and Cognitive Neurology, Vanderbilt University Medical Center, 1161 21st Avenue, A-0118 MCN, Nashville, TN 37232, USA

\section{Key Points}

Seventy-six percent of patients in this large clinical cohort $(n=91)$ had improvement in psychosis on pimavanserin, while $22 \%$ of patients reported side effects.

Positive response to pimavanserin did not differ based on diagnosis, presence of dementia, delusions, use of deep brain stimulation, or prior antipsychotic failure.

Pimavanserin was successfully used with and without other concomitant antipsychotics. At current follow-up, most patients still taking pimavanserin $(n=50)$ are on monotherapy for Parkinson's disease psychosis (66\%), while the remainder of patients (34\%) are on dual therapy, most commonly with quetiapine. 
and delusions are also encountered. Treatment of PD psychosis presents a challenge: dopaminergic therapies, such as levodopa or dopamine agonists, are commonly used for the treatment of motor symptoms of PD, but can exacerbate psychosis. Inversely, typical antipsychotics prescribed for psychotic symptoms may worsen motor symptoms as a result of dopaminergic antagonism, resulting in substantial morbidity [1].

Quetiapine, an atypical antipsychotic, is often used for PD psychosis symptoms in clinical practice, but efficacy has not been consistently shown in clinical trials [4]. Clozapine has previously been shown to have adequate efficacy and safety data for PD psychosis [5, 6], but is infrequently prescribed, because of the risk for agranulocytosis and inconvenient monitoring requirements [1]. Pimavanserin, a selective serotonin $5-\mathrm{HT}_{2 \mathrm{~A}}$ receptor inverse agonist, was recently approved by the US Food and Drug Administration (FDA) to treat PD psychosis [7]. Pimavanserin has fewer adverse effects than antipsychotics that target dopamine receptors, and this is likely due to its selectivity to the $5-\mathrm{HT}_{2 \mathrm{~A}}$ serotonin receptor [8]. However, like all antipsychotics, it carries a black box warning for increased risk of death in elderly demented patients [8]. PD and dementia with Lewy bodies (DLB) are heterogeneous disorders with overlapping features related to the presence of Lewy body inclusions. Historically, pimavanserin has been studied in patients with PD psychosis, but the common pathology of PD and DLB suggest potential efficacy of pimavanserin in patients with DLB psychosis.

To date, reports of real-world experience with pimavanserin have been limited by small sample sizes $(n=2$ and $15)[9,10]$ or limited scope [11], preventing a meaningful analysis of real-world efficacy and patient characteristics, including PD or DLB diagnosis, presence and severity of dementia, deep brain stimulation (DBS) use, and prior antipsychotic failure. In this retrospective chart review, we sought to evaluate the clinical efficacy of pimavanserin in a larger cohort and describe prescribing patterns, concomitant antipsychotic use, titration strategies, and tolerability in patients with various clinical presentations.

\section{Methods}

\subsection{Chart Review}

The study was approved through the Vanderbilt University Medical Center (VUMC) Institutional Review Board, located in the southeast United States. Study data were collected and managed using REDCap electronic data capture tools hosted at VUMC [12].
Prescriptions for pimavanserin at VUMC are processed by the Vanderbilt Specialty Pharmacy, allowing identification of those patients where there was an 'intention to treat.' Medical records were reviewed to confirm the provider's intention to start pimavanserin and assess clinical follow-up. Patients who began pimavanserin treatment were included in the main efficacy analysis, while patients where pimavanserin was prescribed but not started were used as a control sample to assess safety.

We recorded pertinent demographic and clinical information. Demographic details included age, race, and gender. Clinical information included diagnosis, use of DBS, presence and severity of dementia, previous antipsychotic treatments, and clinical indication for switching to pimavanserin, including presence of hallucinations or delusions or both. Prescribing data included date of pimavanserin prescription, method of conversion to pimavanserin if a patient was on concomitant antipsychotic treatment, clinician impression of tolerability and efficacy, clinical indication for discontinuation (if applicable), and subsequent adjunctive or alternative antipsychotic use. Efficacy was defined as a clinically significant improvement in psychosis symptoms after taking pimavanserin for at least 6 weeks - the length of time used to evaluate clinical efficacy in clinical trials [13].

Similar to prior retrospective reviews of dementia in PD and DLB $[14,15]$, dementia in our study was defined as modest-to-major cognitive decline in at least one cognitive domain, noted in the medical record, consistent with Diagnostic and Statistical Manual of Mental Disorders, Fifth Edition (DSM-5) criteria for a mild or major neurocognitive disorder [16]. Patients with total caregiver dependence due to cognitive dysfunction were classified as having severe dementia, and these patients were further separated from those with mild and moderate dementia for our analysis. Total caregiver dependence included needing assistance for all activities of daily living or being unable to meaningfully contribute to the history during a clinic visit.

\subsection{Statistical Analysis}

We used Chi-squared tests to look for statistically significant differences in baseline characteristics and mortality in the pimavanserin treatment patients versus those who did not start pimavanserin. For patients who did start pimavanserin, we used Chi-squared tests to compare the clinical response in patients with DBS, delusions, dementia, and prior and concurrent use of other antipsychotics. Two-tailed confidence intervals for statistical significance were set at 0.05 . IBM SPSS version 24 was used to perform the analyses. 


\section{Results}

\subsection{Baseline Characteristics}

We identified 107 patients who were prescribed pimavanserin by 13 providers at our institution between May 2016 and July 2018 (Fig. 1). All patients were prescribed the recommended $34 \mathrm{mg}$ daily dosage, though two patients later switched to $17 \mathrm{mg}$ daily or every other day because of adverse effects at $34 \mathrm{mg}$.

Of the 91 patients who began pimavanserin treatment, most were male (70/91, 77\%) and Caucasian (86/91, 95\%), with a mean age of 71 years \pm 13 (Table 1$)$. Most (71/91, $78 \%$ ) were diagnosed with $\mathrm{PD}$, while $11(12 \%)$ were diagnosed with DLB, and the remaining nine (10\%) carried diagnoses of PD with early cognitive symptoms $(n=7)$, PD with logopenic aphasia $(n=1)$, or PD with concern of normal pressure hydrocephalus $(n=1)$. For analysis, these nine were included in the PD group. Dementia (68/91, 75\%) and presence of DBS $(27 / 91,30 \%)$ were common in this cohort. Most patients with dementia had mild to moderate symptoms $(52 / 68,76.5 \%)$, while a smaller proportion $(16 / 68$, $23.5 \%$ ) had severe dementia. In the 91 treated patients, at time of treatment initiation, 40 patients (44\%) had only visual hallucinations, while an additional 49 (54\%) had both visual hallucinations and delusions. Only two patients (2\%) were prescribed pimavanserin to treat delusions without accompanying visual hallucinations.

Two-thirds of patients $(58 / 91,64 \%)$ had persistent psychotic symptoms at the time of pimavanserin prescription despite prior treatment with one or more other antipsychotics. Of those, $90 \%$ (52/58) were previously prescribed quetiapine, while $21 \%(12 / 58)$ were prescribed clozapine. Risperidone, haloperidol, asenapine, and olanzapine were each prescribed in three or fewer patients.

Table 1 Baseline characteristics

\begin{tabular}{ll}
\hline Characteristic & Value \\
\hline Age (years), mean \pm standard deviation & $70.8 \pm 12.9$ \\
Sex, $n(\%)$ & \\
Male & $70(77)$ \\
Female & $21(23)$ \\
Diagnosis, $n(\%)$ & \\
Parkinson's disease & $80(88)$ \\
Dementia with Lewy bodies & $11(12)$ \\
Deep brain stimulation, $n(\%)$ & \\
Yes & $27(30)$ \\
No & $63(69)$ \\
Other (deep brain stimulation removed) & $1(1)$ \\
Symptom presence, $n(\%)$ & \\
Hallucinations & $89(98)$ \\
Delusions & $51(56)$ \\
Dementia & $68(75)$ \\
Previous antipsychotic trial, $n(\%)$ & \\
Yes & $58(64)$ \\
No & $33(36)$ \\
Antipsychotic(s) tried previously, $n(\%)$ & \\
Quetiapine & $52(57)$ \\
Clozapine & $12(13)$ \\
Other & $10(11)$ \\
\hline
\end{tabular}

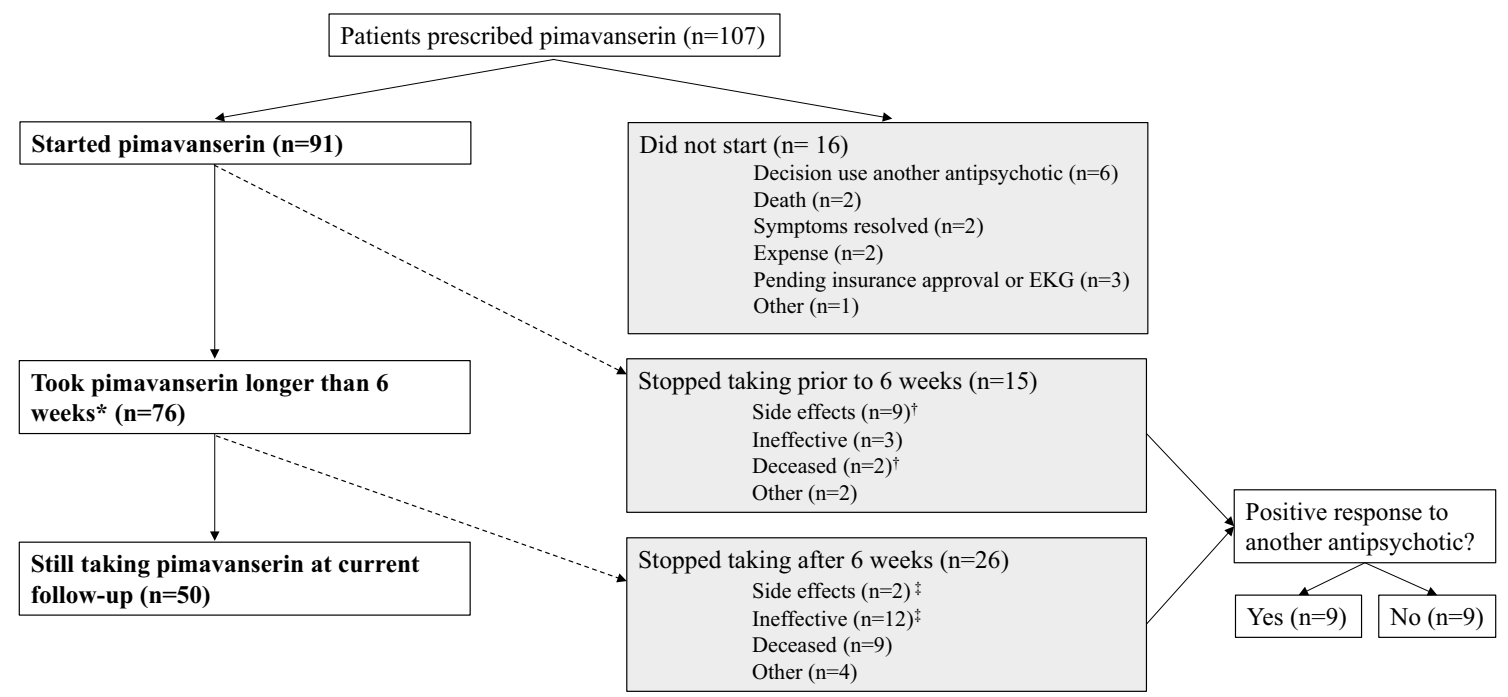

*includes 1 patient who continued on pimavanserin but had only been on therapy for 4 weeks at time of data collection tincludes 1 patient whose caregiver reported both side effects and patient death as reasons for stopping tincludes 1 patient who reported stopping both due to side effects and lack of efficacy

Fig. 1 Depiction of treatment outcomes in patients prescribed pimivanserin. $E K G$ electrocardiogram 


\subsection{Titration Strategies}

We sought to understand how providers advised patients to start pimavanserin treatment, particularly in the setting of past antipsychotic use. Approximately one-third of patients $(33 / 91,36 \%)$ began treatment de novo. For those with previous antipsychotic failure $(n=58), 21$ patients $(36 \%)$ had stopped or were advised to stop their other antipsychotic prior to pimavanserin initiation. Fifteen patients $(26 \%)$ were advised to taper off their other antipsychotic after starting pimavanserin, and the duration of this taper ranged from 1 week to 3 months. Twenty-two patients (38\%) were advised to continue current antipsychotic treatment in addition to pimavanserin.

\subsection{Response to Pimavanserin}

Sixty-nine of the 91 treated patients (76\%) had decreased psychosis symptoms after starting pimavanserin therapy. Four of these 69 patients (6\%) eventually discontinued pimavanserin because efficacy was not sustained. No statistically significant differences in efficacy were found according to previous antipsychotic use $(p=0.6)$, DBS status $(p=0.39)$, presence of any dementia $(p=0.8)$, or delusions $(p=0.41)$. Those with mild-moderate dementia responded no differently than those with severe dementia $(p=0.61)$, though severe dementia was associated with increased mortality compared to mild-moderate dementia $\left(\chi^{2}=5.67, p=0.02\right)$. We found no difference in pimavanserin response in patients with just hallucinations as compared to those with both hallucinations and delusions $(p=0.35)$; nor did we see a differential response in patients with $\mathrm{PD}$ psychosis versus those with DLB psychosis $(p=0.54)$.

\subsection{Current Patient Status and Concomitant Antipsychotic Use}

At the latest follow-up, 50 patients of the 77 living patients $(65 \%)$ remain on pimavanserin, with a mean treatment duration of $14.6 \pm 6.7$ months. The 27 living patients no longer on treatment stopped pimavanserin primarily because of a lack of efficacy $(11 / 77,14 \%)$, side effects $(9 / 77,12 \%)$, or both $(1 / 77,1 \%)$. Six patients $(8 \%)$ stopped for reasons unrelated to medication effects, including the desire to reduce overall medication burden and negative media reporting on pimavanserin.

Two-thirds of patients who remain on pimavanserin $(33 / 50,66 \%)$, as at the latest follow-up, receive pimavanserin monotherapy, and just over half of these monotherapy patients $(17 / 33,52 \%)$ were able to stop use of another antipsychotic. Seventeen patients currently taking pimavanserin $(17 / 50,34 \%)$ are on dual therapy with one or more other antipsychotics [quetiapine $=16$ (median daily dose $50 \mathrm{mg}$ ); olanzapine $=1(2.5 \mathrm{mg}$ as needed $)$; clozapine $=1$ (daily dose $50 \mathrm{mg}$ )]. Of the patients without prior antipsychotic use who are still taking pimavanserin $(20 / 50,40 \%)$, four $(20 \%)$ required addition of a second antipsychotic with pimavanserin for adequate control of psychosis.

Those currently on concomitant quetiapine (16/50, 32\%) typically dose this at night $(10 / 16,63 \%)$, while several take divided daily doses of quetiapine, but take a higher dose at night $(4 / 16,25 \%)$. This tendency for nighttime dosing appears to be more related to avoidance of daytime sedation than to improvement of nighttime-specific symptoms like increased nocturnal psychosis or insomnia, though this conclusion is based on general impressions of provider documentation. Ten of the patients currently on concomitant pimavanserin and quetiapine $(10 / 16,63 \%)$ required an increase in the quetiapine dose at some point after starting pimavanserin. These doses were increased due to increases in psychosis with disease progression. The average length of pimavanserin therapy in patients on concomitant quetiapine is $16 \pm 7.7$ months.

\subsection{Tolerability of Pimavanserin and Mortality Data}

Twenty of the 91 treated patients (22\%) had adverse effects possibly related to pimavanserin treatment. The most commonly reported adverse effects were worsening gait instability $(5 / 91,5 \%)$, somnolence $(3 / 91,3 \%)$, mild lower extremity edema $(2 / 91,2 \%)$, nausea $(2 / 91,2 \%)$, and nightmares $(2 / 91$, $2 \%)$. Eleven patients (12\%) stopped taking pimavanserin due to adverse effects. One patient who reported sedation was able to tolerate pimavanserin at a lower dose $(17 \mathrm{mg}$ daily), with effective reduction in psychosis. Another patient changed dosing to $17 \mathrm{mg}$ every other day because of concern about adverse events as reported in the media, though that patient had not reported any side effects at the original dosage of $34 \mathrm{mg}$ daily. These were the only patients (2/91, $2 \%$ ) taking doses other than the recommended daily dose of $34 \mathrm{mg}$.

QTc prolongation is a potential risk of antipsychotic therapy [17], and one patient (1\%) with premorbid heart disease died from suspected cardiac arrest. No other acute cardiac events were reported.

Death occurred in $15 \%$ (14/91) of patients started on pimavanserin versus $44 \%$ (7/16) of patients prescribed but not started on pimavanserin $\left(\chi^{2}=6.94, p<0.01\right)$.

\subsection{Success Rate with Alternate Antipsychotics}

Of the 18 patients (20\%) who stopped pimavanserin and tried an alternate antipsychotic, only nine (50\%) had success with the alternate therapy (four with quetiapine, four with clozapine, and one with olanzapine). Six of these 18 
patients (33\%) had been unable to tolerate pimavanserin, out of whom five (83\%) had success with another antipsychotic. Eight patients of the 18 who tried an alternate antipsychotic (44\%) had stopped pimavanserin because of a complete lack of clinical response, and three of these eight (38\%) had success with another antipsychotic. The remaining four patients of the 18 who tried an alternate antipsychotic (22\%) are those who had an initial improvement on pimavanserin that was not sustained. Of these, three patients (75\%) had success with another antipsychotic.

Of the 15 patients who stopped pimavanserin after taking it for less than 6 weeks (recommended time to efficacy), seven $(47 \%)$ did not try an alternate antipsychotic. Three of those who did try another antipsychotic $(3 / 8,38 \%)$ had success with the alternate agent, while the rest were either unable to tolerate the medication $(3 / 8,38 \%)$ or had no clinical improvement $(2 / 8,25 \%)$.

\subsection{Non-initiators: Prescribed but Not Filled}

For those who did not begin pimavanserin treatment $(n=16)$, reasons included cost $(2 / 16,13 \%)$, pending insurance approval or delay while waiting for a baseline electrocardiogram (EKG) $(3 / 16,19 \%)$, resolution of symptoms (2/16, $13 \%)$, decision to continue or start a different antipsychotic $(6 / 16,38 \%)$, and death $(2 / 16,13 \%)$. One patient did not start pimavanserin because of caregiver concern about side effects, unrelated to negative media reporting. Demographic characteristics, age (71.4 \pm 8.1 years), gender, diagnosis, and reported psychiatric symptoms were similar to those patients who did begin pimavanserin treatment.

\section{Discussion}

This is the largest study to date assessing the real-world clinical efficacy of pimavanserin for PD-related psychosis, and we also considered other important clinical characteristics. Our findings are consistent with controlled trials and smaller case reports, as pimavanserin was tolerated and effective in reducing psychotic symptoms in most patients. Efficacy was similar regardless of diagnosis, presence of dementia, DBS use, or prior antipsychotic exposure and response.

\subsection{General Efficacy}

As reported in recent case series of pimavanserin, despite incomplete resolution of psychosis, the clinical improvements support its continued use [9]. In this chart review, one patient was so pleased with his response he reported that taking pimavanserin was "like waving a magic wand." A caregiver for a different patient reported that she "wouldn't want to go without it" because improvement in her husband's psychosis was so striking. This improvement is consistent with data from the pivotal study in PD psychosis where reduction in psychotic symptoms was clinically meaningful, and complete resolution of psychosis was not the primary study aim [13]. Clinically, these improvements can be satisfying to the provider, caregiver, and patient.

\subsection{Efficacy of Pimavanserin in Patients with Dementia and DBS}

Psychosis is more common in patients with dementia and is also a risk factor for the development of dementia [18-24]. Clinical trials for pimavanserin did not explicitly exclude patients on the basis of a diagnosis of dementia, but required a Mini Mental State Examination (MMSE) cut-off score of $>21$ for inclusion [13]. In our experience, we find equivalent efficacy for improvement in psychosis regardless of the presence and severity of dementia, suggesting that pimavanserin can be an effective treatment of psychosis in patients with DLB and PD dementia. Future trials in DLB and PD dementia are needed to test this hypothesis.

While the pivotal study included ten patients with DBS, it did not explicitly test for response differences in this subset [13]. We find similar efficacy in a larger sample $(n=27)$ of patients with DBS, suggesting that pimavanserin can be effective in these patients.

\subsection{Safety/Mortality}

The most common adverse effect seen in our cohort was gait instability. This is consistent with previous experience, where falls were more frequently seen in pimavanserin (11\%) versus the placebo (9\%) arm [13]. As noted, we did not see increased mortality rates in the group that started pimavanserin compared to those who did not start the medication. There has been recent discussion on whether patients taking pimavanserin carry a higher mortality risk [25], and our real-world data does not support this assertion, in keeping with other real-world reports [11]. However, this retrospective study was not powered to make claims on mortality risk with pimavanserin, and we report our clinical findings with this important caveat. Of note, two patients in our cohort chose to self-discontinue or reduce their dose of pimavanserin because of negative media reporting, despite symptomatic improvement with pimavanserin and no reported side effects. Hallucinations or visual disturbance recurred for both patients.

We believe that due to the increased incidence of psychosis in later stages of PD, patients starting pimavanserin 
are likely older, with more medical comorbidities and a greater likelihood of mortality. Accounting for other variables like swallowing difficulty, falls, and rates of pneumonia and urinary tract infections may negate the mortality signal reported in the general media. Such factors may have similarly affected the mortality rate in our pimavanserin non-starter group; thus we again advise caution in interpreting the clinical significance of group mortality comparisons in the present study.

\subsection{Concomitant Antipsychotic Use and Recommendations}

Our finding that many patients tolerate concomitant quetiapine with pimavanserin is consistent with previous reports, where $17 \%$ of patients were exposed to quetiapine within 3 weeks prior to their baseline visit [13]. We propose that pimavanserin is a reasonable first-line treatment for PD or DLB psychosis, and it appears that quetiapine can be added subsequently, at the lowest possible dose, for treatment resistant, residual, or newly emergent psychotic symptoms. There appears to be a substantial proportion of patients who have psychosis symptoms that are difficult to treat. About one-third of our patients currently taking pimavanserin require multiple antipsychotics for adequate control of symptoms $(17 / 50,34 \%)$, and future studies should explore clinical features that predict treatment response. It is possible that higher dosages of pimavanserin may be useful, but our cohort did not go above the $34 \mathrm{mg}$ daily dosage.

\subsection{Limitations}

Limitations of this study include its retrospective design and reliance on prescriber documentation of treatment efficacy and outcomes. The 13 providers who prescribed and documented clinical follow-up did not use a singular outcome measure, such as Clinical Global Impression (CGI). Thus, we relied on documented clinical impressions based on provider reports. Another important limitation is potential selection bias; those patients who have not tried pimavanserin because of adequate response to other antipsychotics were not included. This leaves out a cohort of patients on other antipsychotics who might (1) have better or equal response to pimavanserin or (2) not respond to pimavanserin. Another limitation is the small size of our "non-starter" group, which we used as a control group for comparison in analysis. Additionally, our sample lacks diversity in terms of gender and race. We also could not ascertain differences related to specific types of psychotic symptoms beyond classification of hallucinations and delusions (e.g., tactile versus visual hallucinations and delusional misidentifications). Variations in neurobiological determinants of psychotic symptoms [26, 27] could account for a differential response to pimavanserin; thus future prospective studies should differentiate between types of psychosis.

Another important limitation of our study was precise classification of dementia severity. Our definition of dementia was consistent with prior studies, but because of inconsistencies in the documentation noted above, we could only separate the most severely demented patients from other, less demented patients for analysis. We would have preferred to differentiate between mild cognitive impairment and mild, moderate, and severe dementia, as distinctions in clinical presentation of these patients are relevant to providers making treatment decisions. However, because we did not see differences in efficacy in patients with and without dementia, nor with mild-moderate versus severe dementia, we do not suspect that our results would have changed were we able to make this distinction. This assertion would be best confirmed in a prospective study using the same, validated tool to measure cognitive function in each patient.

\section{Conclusions}

In conclusion, we describe clinical efficacy of pimavanserin in PD-related psychosis in a large $(n=91)$ sample of patients from a tertiary referral academic medical center. Our results are consistent with those reported in clinical trial experience [13]. Most patients tolerated and reported clinical improvement on pimavanserin, regardless of diagnosis (PD versus DLB), prior antipsychotic use, dementia, and DBS status. Although the majority of patients remain on pimavanserin monotherapy, many patients responded well to dual therapy with pimavanserin and quetiapine. Finally, half of patients with no clinical response to pimavanserin after 6 weeks also responded poorly to subsequent trials of other antipsychotic medications $(3 / 6,50 \%)$, suggesting a group of medically refractory patients that may have a different underlying biological substrate. Based on these data, we recommend pimavanserin as first-line treatment for PD-related psychosis because of the excellent efficacy and tolerability observed in our patients.

\section{Compliance with Ethical Standards}

The study was approved through the Vanderbilt University Medical Center (VUMC) Institutional Review Board.

Funding No funding was received for the conduct or reporting of this research.

Conflict of interest Jessie Sellers has received research support and personal fees for speaker bureau participation from Teva Neuroscience. Dr. Darby is supported by grants from the Alzheimer's Asso- 
ciation, Brightfocus Foundation, and Vanderbilt Institute for Clinical and Translational Research. Daniel O. Claassen receives research support from the National Institute of Neurological Disorders and Stroke (NINDS), Griffin Foundation, Michael J. Fox Foundation, and Huntington's Disease Society of America (HDSA), and pharmaceutical grant support from AbbVie, Acadia, Biogen, BMS, Cerecour, Eli Lilly, Lundbeck, Jazz Pharmaceuticals, Teva Neuroscience, Wave Life Sciences, and Vaccinex. He has received personal fees for consulting and advisory board participation from Acadia, Adamas, Lundbeck, Neurocrine, and Teva Neuroscience. Given a potential conflict with Acadia (makers of pimavanserin), he excluded himself from data acquisition and data analysis, but provided direction on project design, and critical review and revision of the manuscript. Alma Farooque has no conflicts of interest to disclose.

Open Access This article is distributed under the terms of the Creative Commons Attribution-NonCommercial 4.0 International License (http://creativecommons.org/licenses/by-nc/4.0/), which permits any noncommercial use, distribution, and reproduction in any medium, provided you give appropriate credit to the original author(s) and the source, provide a link to the Creative Commons license, and indicate if changes were made.

\section{References}

1. Zahodne LB, Fernandez HH. Pathophysiology and treatment of psychosis in Parkinson's disease: a review. Drugs Aging. 2008;25(8):665-82.

2. Fénelon G, Soulas T, Zenasni F, De Langavant LC. The changing face of Parkinson's disease-associated psychosis: a cross-sectional study based on the new NINDS-NIMH criteria. Mov Disord. 2010;25(6):755-9. https://doi.org/10.1002/mds.22839.

3. Aarsland D, Ballard C, Larsen JP, McKeith I. A comparative study of psychiatric symptoms in dementia with Lewy bodies and Parkinson's disease with and without dementia. Int J Geriatr Psychiatry. 2001;16(5):528-36.

4. Shotbolt P, Samuel M, David A. Quetiapine in the treatment of psychosis in Parkinson's disease. Ther Adv Neurol Disord. 2010;3:339-50.

5. Parkinson Study Group. Low-dose clozapine for the treatment of drug-induced psychosis in Parkinson's disease. N Engl J Med. 1999;340(10):757-63.

6. Pollak P, Tison F, Rascol O, et al. Clozapine in drug induced psychosis in Parkinson's disease: a randomised, placebo controlled study with open follow up. J Neurol Neurosurg Psychiatry. 2004;75(5):689-95.

7. Mathis MV, Muoio BM, Andreason P, et al. The US Food and Drug Administration's perspective on the new antipsychotic pimavanserin. J Clin Psychiatry. 2017. https://doi.org/10.4088/ jcp.16r11119.

8. Yuan M, Sperry L, Malhado-Chang N, et al. Atypical antipsychotic therapy in Parkinson's disease psychosis: a retrospective study. Brain Behav. 2017;7(6):e00639. https://doi.org/10.1002/ brb3.639.

9. Friedman JH. A retrospective study of pimavanserin use in a movement disorders clinic. Clin Neuropharmacol. 2017;40(4):157-9.

10. Hermanowicz N. Delusional misidentification in Parkinson's disease: report of two cases and a review. Postgrad Med. 2018;130(2):280-3.
11. Moreno GM, Gandhi R, Lessig SL, Wright B, Litvan I, Nahab FB. Mortality in patients with Parkinson disease psychosis receiving pimavanserin and quetiapine. Neurology. 2018;00:1-3. https://doi. org/10.1212/WNL.0000000000006396.

12. Harris PA, Taylor R, Thielke R, Payne J, Gonzalez N, Conde JG. Research electronic data capture (REDCap) - a metadata-driven methodology and workflow process for providing translational research informatics support. J Biomed Inform. 2009;42(2):377-81.

13. Cummings J, Isaacson S, Mills R, et al. Pimavanserin for patients with Parkinson's disease psychosis: a randomised, placebo-controlled phase 3 trial. Lancet. 2014;383(9916):533-40.

14. Rana AQ, Yousuf MS, Naz S, Qaaty N. Prevalence and relation of dementia to various factors in Parkinson's disease. Psychiatry Clin Neurosci. 2012;66(1):64-8. https://doi.org/10.111 1/j.1440-1819.2011.02291.x.

15. Savica R, Grossardt BR, Bower JH, Boeve BF, Ahlskog JE, Rocca WA. Incidence of dementia with Lewy bodies and Parkinson disease dementia. JAMA Neurol. 2013;70(11):1396-402. https://doi. org/10.1001/jamaneurol.2013.3579.

16. (2013) Diagnostic and statistical manual of mental disorders: DSM-5. American Psychiatric Publishing, Arlington.

17. Beach SR, Celano CM, Sugrue AM, Adams C, Ackerman MJ, Noseworthy PA, Huffman JC. QT prolongation, torsades de pointes, and psychotropic medications: a 5-year update. Psychosomatics. 2018;59(2):105-22. https://doi.org/10.1016/j. psym.2017.10.009.

18. Gasca-Salas C, Clavero P, García-García D, Obeso JA, RodríguezOroz MC. Significance of visual hallucinations and cerebral hypometabolism in the risk of dementia in Parkinson's disease patients with mild cognitive impairment. Hum Brain Mapp. 2016;37(3):968-77.

19. Aarsland D, Andersen K, Larsen JP, Lolk A, Kragh-Sørensen P. Prevalence and characteristics of dementia in Parkinson disease: an 8-year prospective study. Arch Neurol. 2003;60(3):387-92.

20. Anang JBM, Gagnon JF, Bertrand JA, et al. Predictors of dementia in Parkinson disease: a prospective cohort study. Neurology. 2014;83(14):1253-60.

21. Uc EY, McDermott MP, Marder KS, et al. Incidence of and risk factors for cognitive impairment in an early Parkinson disease clinical trial cohort. Neurology. 2009;73(18):1469-77.

22. Morgante L, Colosimo C, Antonini A, et al. Psychosis associated to Parkinson's disease in the early stages: relevance of cognitive decline and depression. J Neurol Neurosurg Psychiatry. 2012;83(1):76-82.

23. Ibarretxe-Bilbao N, Ramirez-Ruiz B, Junque C, et al. Differential progression of brain atrophy in Parkinson's disease with and without visual hallucinations. J Neurol Neurosurg Psychiatry. 2010;81(6):650-7.

24. Ramirez-Ruiz B, Junque C, Marti M-J, Valldeoriola F, Tolosa E. Cognitive changes in Parkinson's disease patients with visual hallucinations. Dement Geriatr Cogn Disord. 2007;23(5):281-8.

25. Ellis B, Hicken M (2018) FDA worried drug was risky; now reports of deaths spark concern. CNN. https://www.cnn. com/2018/04/09/health/parkinsons-drug-nuplazid-invs/index .html. Accessed 18 Aug 2018.

26. Darby RR, Laganiere S, Pascual-Leone A, Prasad S, Fox MD. Finding the imposter: brain connectivity of lesions causing delusional misidentifications. Brain. 2017;140(2):497-507.

27. Darby R, Prasad S. Lesion-related delusional misidentification syndromes: a comprehensive review of reported cases. J Neuropsychiatry Clin Neurosci. 2016;28(3):217-22. 